\title{
Interactive Image Retrieval Using Smoothed Nearest Neighbor Estimates ${ }^{\star}$
}

\author{
Miguel Arevalillo-Herráez and Francesc J. Ferri \\ Departament d'Informàtica, Universitat de València, \\ Av. Vicent Andrés-Estellés, s/n, 46100 Burjassot (València), Spain \\ \{miguel.arevalillo,francesc.ferri\}@uv.es
}

\begin{abstract}
Relevance feedback has been adopted by most recent Content Based Image Retrieval systems to reduce the semantic gap that exists between the subjective similarity among images and the similarity measures computed in a given feature space. Distance-based relevance feedback using nearest neighbors has been recently presented as a good tradeoff between simplicity and performance. In this paper, we analyse some shortages of this technique and propose alternatives that help improving the efficiency of the method in terms of the retrieval precision achieved. The resulting method has been evaluated on several repositories which use different feature sets. The results have been compared to those obtained by the nearest neighbor approach in its standard form, suggesting a better performance.
\end{abstract}

Keywords: CBIR, image retrieval framework, relevance feedback.

\section{Introduction}

Content based image retrieval (CBIR) embraces a set of techniques which aim to recover pictures from large image repositories according to the interests of the user. Usually, a CBIR system represents each image in the repository as a set of features (usually related to color, texture and shape), and uses a set of distance functions defined over this feature space to estimate similarity between pictures. In this context, a query is usually composed of one or more sample pictures, and the task of the CBIR system is to retrieve the set of images which best matches this query. Indeed, the performance of such a system depends on both the feature space and the distance function used to estimate the similarity between pictures. In this direction, a large number of features and distance functions have been proposed in the past [123].

The assumption that subjective or semantic similarity is related to the similarity between low level features is implicit to this way of posing the retrieval problem. But since this does not hold true, the goal of most CBIR techniques is to reduce the existing gap between the semantics induced from the low level

\footnotetext{
* Work partially funded by Feder, GV and MEC through projects TIN2009-14205C04-03, ACOMP/2010/287 and Consolider Ingenio 2010 CSD2007-00018.
} 
features and the real high level meaningful semantics of the image. Relevance feedback has been adopted by most recent CBIR systems to reduce the so-called semantic gap 44. When relevance feedback is used, the search is considered an iterative process in which the original query is refined interactively, to progressively obtain a more accurate result. At each iteration, the system retrieves a series of images according to a pre-defined similarity measure, and requires user interaction to mark the relevant and non relevant retrievals. This data is used to modify some system parameters and produce a new set of results, repeating the process until a satisfying enough result is obtained. In this context, the relationship between any image in the database and the user's desire is usually expressed in terms of a relevance value, which is aimed at directly reflecting the interest the user may have in the image and is to be refined at each iteration.

A large amount of relevance feedback algorithms use the user's selection to search for global properties which are shared by the relevant samples available at each iteration 4. From a Pattern Recognition viewpoint, this can be seen as obtaining an appropriate estimate of the probability of (subjective) relevance. Many different approaches exist to model and progressively refine these estimates. But taking into account that this constitutes a small sample problem whose models cannot be reliably established because of the semantic gap, nonparametric distance-based methods using neighbors are particularly appealing in this context and may constitute an appropriate trade off [56]7. The main idea in these methods is to assess relevance using distances to relevant and nonrelevant neighbors of a given image. In this paper, we consider several already proposed algorithms and analyse their behavior to identify some major pitfalls and motivatedly arrive at several improvements to the original algorithms.

The remainder of the paper is organized as follows. The next section presents the model used, outlines the assumptions made, and presents the name conventions used throughout the article. Then, the nearest neighbor approach is outlined. After, some problems related to the application of this technique in the field of CBIR are analysed. Later, some improvements that tackle these problems are introduced, proposing an alternative formulation of the approach. Finally, the resulting algorithm is compared to the original one [5,6] and some final conclusions are drawn.

\section{Relevance-Guided Interactive Image Retrieval}

Let us assume we have a repository of images $\mathcal{X}=\left\{x_{1}, \cdots, x_{m}\right\}$ conveniently represented in a metric feature space, $\mathcal{F}$, whose associate distance measure is $d: \mathcal{F} \times \mathcal{F} \longrightarrow \mathbb{R}^{\geq 0}$. Usually, in the image retrieval context, the representation space is assumed to be the $D$-dimensional space $\mathbb{R}^{D}$, which may embrace multiple low level descriptors (e.g. color, texture or shape) and the distance $d$ is constructed by combination from simple distance measures over each descriptor [1].

Let us assume that a particular user is interested in retrieving images from $\mathcal{X}$ related to a particular semantic concept. The user's interest can be modelled in 
the feature space as a probability function, $p($ relevant $\mid x)$, which carries in fact a hidden dependence on the given repository, $\mathcal{X}$.

Single point query approaches assume that this probability function can be appropriately represented by a single (ideal) point $c \in \mathcal{F}$ possibly along with a convenient axis or feature reweighting [8]. This approach can be extended to use a set of representative points $\mathcal{C}=\left\{c_{1} \cdots c_{\ell}\right\}$ as in typical multipoint query setups 9 .

The goal of the retrieval system at each relevance feedback iteration is to find a set of images from $\mathcal{X}$ that contains as much relevant images as possible using the available information. Single point methods use a distance measure to rank images while multiple point methods usually (linearly) combine rankings to each representative in $\mathcal{C}$.

The available information or user feedback is given by the set of images from $\mathcal{Q} \subset \mathcal{X}$ already seen by the user and marked either as relevant (positive), $\mathcal{Q}^{+}$or as non-relevant (negative), $\mathcal{Q}^{-}$. Both disjoint subsets, $\mathcal{Q}^{+}$and $\mathcal{Q}^{-}$, can be seen as samples corresponding to the distribution functions that determine $p$ (relevant $\mid x)$ as in a typical two-class classification setting. The problem is that these samples are far from being truly representative both because of the small sample size case and the strong dependences introduced by the way in which new evidence is progressively taken into account.

\section{Nearest Neighbor (NN) Approach}

Nearest neighbors methods have been extensively used in the context of learning, vision and pattern recognition due to their well-known, convenient and well studied practical and asymptotic behavior [1011].

In particular, the ratio of fraction of neighbors of a certain kind to the volume of the hypersphere containing them is known to be a good estimate of the corresponding probability distributions 12 . This fact has been used in the context of image retrieval [7] in the particular case of a single nearest neighbor to obtain estimates for the relevant and non-relevant classes as inversely proportional to the volume of the corresponding 1-Neighborhoods, $V_{R}\left(d_{R}(x)\right)$ and $V_{N}\left(d_{N}(x)\right)$, where the subscripts refer to the nearest relevant $(\mathrm{R})$ and non-relevant $(\mathrm{N})$ neighbors, respectively; and $d_{R}$ and $d_{N}$ are the corresponding distances to each neighbor from $x$.

From the separate estimates and obviating the exponent $D$ and some constant terms in the volume formulae, the following expression can be arrived at [7:

$$
p(\text { relevant } \mid x)=\frac{d_{N}(x)}{d_{R}(x)+d_{N}(x)}
$$

A simplified version of this estimate already used in this context [5] is given by:

$$
p(\text { relevant } \mid x) \propto \frac{d_{N}(x)}{d_{R}(x)}
$$

where the symbol $\propto$ indicates that a convenient nondecreasing mapping is to be used. On the other hand, it is worth noting that in the image retrieval context 
what is in fact important is the ranking these estimates induce and not their absolute values. For convenience, normalized relevance scores are used in practice as e.g. $R(x)=1-e^{\frac{d_{N}(x)}{d_{R}(x)}}$ [5]. Note that all estimates above whether normalized or not, give rise to the same ranking and consequently are equivalent from the point of view of relevance feedback.

\section{Considerations about NN Estimates in CBIR}

Several problems arise when applying NN estimates in the context image retrieval with relevance feedback. A first problem already reported in [7] comes from the relative sizes of the $\mathcal{Q}^{+}$and $\mathcal{Q}^{-}$sets. In general, the number of relevant items is by far smaller than the number of non-relevant ones, even in the surroundings of the elements in $\mathcal{C}$. This causes that typically the number of elements in $\mathcal{Q}^{+}$be also lower than in $\mathcal{Q}^{-}$. When a relevant selection is surrounded by non-relevant ones, the above rankings produce high values in a very small closed region around it. But from the images outside this region, the top ranked ones are those which are far from both relevant and non-relevant samples. This undesirable effect is illustrated in Figure 1 using the simplest ratio. The chances of this type of situation increase with the relevance feedback iterations, as areas around positive selections tend to be explored more in depth.

Very coupled with the first problem and already identified in [7] is the fact that NN density estimates become very unreliable under the small sample size case. The use of distances to $k$-th neighbors instead of using $k=1$ has already been proposed to obtain (slightly) more stable estimates.

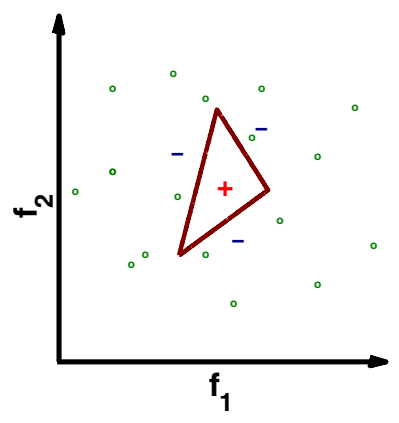

(a)

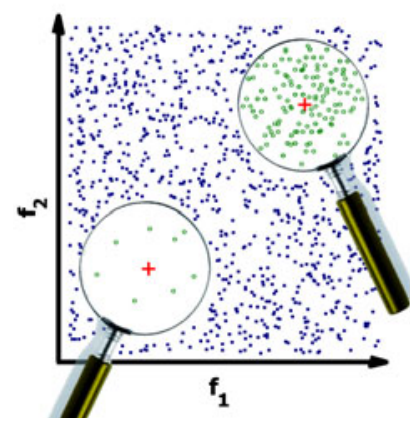

(b)

Fig. 1. Plus signs represent samples that the user has marked as relevant and minus signs those which have been marked as non relevant. Circles represent other existing images. (a) only the pictures within the frontier depicted would yield values of $d_{N}\left(x_{i}\right) / d_{R}\left(x_{i}\right)$ above 1 . Since there are no images in this area, the most relevant samples may be the farthest from positive and negative samples. (b) When images in the repository (circles) are unevenly populated, some regions may dominate rankings. Top ranked images will be all in the close neighborhood of just one of the relevant sample (the one at the top right corner). 
Another important problem not described in previous works is caused by differently populated regions in the feature space. As the ratio of distances is defined in a global way, densely populated regions with high relevance values will tend to dominate the ranking which may result in an undesirable effect in the general case of multipoint query. This problem is caused both by the possibly uneven distribution of images in the repository, $\mathcal{X}$ (as illustrated in Figure 10), and also because of the complex relationship between perceptual similarity and the distance used to find neighbors which may in turn be different in different regions of the feature space. That is, the probability of relevance may scale differently with distance in different regions.

\section{Local Searching Using Smoothed NN Estimates}

Even with the above mentioned problems, NN-based relevance feedback gives surprisingly good results in practice comparable to other state-of-the-art techniques in most practical cases [7. Nevertheless and apart from other improvements related to more meaningful or robust representations (e.g. using dissimilarity spaces) or using hybridization techniques (e.g. with Bayesian relevance feedback), there is still room for improvement in the NN approach to relevance feedback itself.

First, a conveniently smoothed NN estimate can be defined by increasing the importance of $\mathcal{Q}^{+}$. As all previously defined ratios of distances are equivalent with regard to the ranking they induce, we will consider the simple one in Eq. 1. This unnormalized ratio has the advantage of having a simple interpretation in terms of the error rate of the 1-NN classifier [13] and it has also been previously used to derive NN classification rules for imbalanced problems [14].

Using the unnormalized ratio and assuming that the distance to the closest element in $\mathcal{C}$ was available for every picture stored in the repository $\mathcal{X}$, a feasible strategy to smoother estimates would be to introduce a Moderating Term (MT) in Eq. 1. directly related to this distance. As these are not available (the elements in $\mathcal{C}$ are the unknowns of the problem that ideally represent the user's desire), a reasonable approximation is to consider the relevant selections in $\mathcal{Q}^{+}$instead. To this end, the ratio in Eq. [1] is multiplied by the inverse of the distance to the closest relevant sample. In this simple and parameterless way, those points which are close to any of the elements in $\mathcal{Q}^{+}$are rewarded against others which lay farther from them in the feature space. In particular, the following expression is used to compute the relevance scores:

$$
R(x)=1-e^{-\frac{d_{N}(x)}{d_{R}(x)^{2}}}
$$

This expression gives more importance to positive than to negative selections, a consistent approach to deal with the significant differences in the cardinality of the sets of relevant and non relevant selections. The effect of using this smoothed estimate is illustrated in Figure 2.

To deal with the problem of differently populated areas, instead of considering the relevance score, $R(x)$, to produce a global ranking, a set of $r$ local searches 


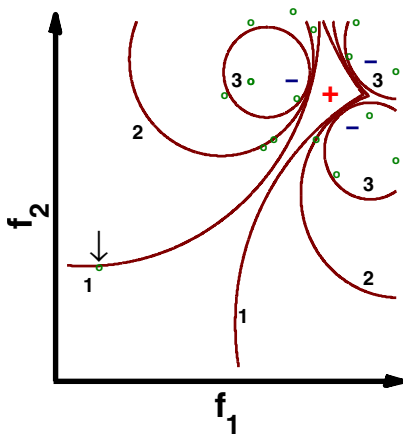

(a)

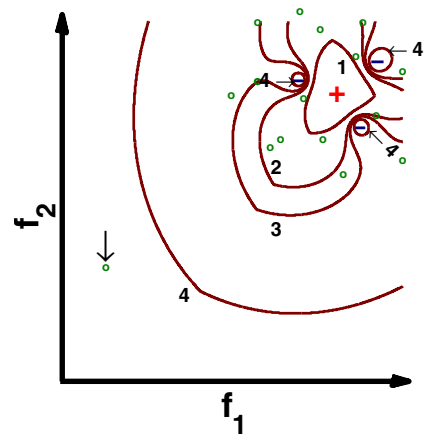

(b)

Fig. 2. Effect of using smoothed and non smoothed estimates. Dots represent images in the repository. Plus and minus signs represent relevant and non relevant selections respectively. The lines are contours of equal score. These have been numbered in decreasing order of relevance. (a) shows these contours using non smoothed estimates; and (b) using smoothed estimates (Eq. 2). In (a), the picture pointed by the arrow would be the closest. In (b) this would be the farthest image of all.

(one per relevant selection $q_{i}^{+}$in the set $\mathcal{Q}^{+}$) will be carried out. Each of these searches is performed using equation 2 , but considering the picture $q_{i}^{+}$as the only relevant sample. This results in a set of $r$ independent rankings $R=\left\{R_{1} \cdots R_{r}\right\}$, one for each local search. Finally, each picture is assigned a score which is inversely proportional to its best ranking position in the set of rankings $R$. This technique makes the approach robust against different density areas.

\section{Empirical Evaluation}

In order to evaluate the impact of the improvements introduced in this work, a comparative analysis of the results obtained with and without them in the original NN approach [5] without any other independent extensions is considered. To evaluate the independent effect of each of the mechanisms proposed we have compared the proposed smoothed NN estimate with local search to those obtained with: (a) the basic NN technique [5]; (b) the NN approach incorporating the local searches (LS) approach; (c) the NN approach adding only the moderation term (MT) technique to handle the surrounding problem. We will refer to these algorithms as the original, the original+LS and the original $+\mathrm{MT}$ respectively.

Exhaustive experimentation has been carried out using three well distinct repositories:

- The commercial collection "Art Explosion", distributed by the company Nova Development. The 10 x 3 HS color histogram and six texture features have been computed for each picture in this database, namely Gabor 
Convolution Energies, Gray Level Co-occurrence Matrix, Gaussian Random Markov Fields, the coefficients of fitting the granulometry distribution with a B-spline basis, and two versions of the Spatial Size distribution, one using a horizontal segment and another with a vertical segment [15].

- The subset of the Corel database used in [5]. This is composed of 30000 images which were manually classified into 71 categories. The descriptors used are those provided in the KDD-UC1 11 repository, namely: A nine component vector with the mean, standard deviation and skewness for each hue, saturation and value in the HSV color space; a 16 component vector with the co-ocurrence in horizontal, vertical and the two diagonal directions; a 32 component vector with the $4 \times 2$ color HS histograms for each of the resulting sub-images after one horizontal and one vertical split; and a 32 component vector with the HS histogram for the entire image.

- A small repository which was intentionally assembled for testing, using some images obtained from the Web and others taken by the authors. The 1508 pictures it contains are classified as belonging to 29 different themes such as flowers, horses, paintings, skies, textures, ceramic tiles, buildings, clouds, trees, etc. In this case, the descriptors include a $10 \times 3$ HS color histogram and texture information in the form of two granulometric cumulative distribution functions [15].

The distances between features have been estimated using the histogram intersection [16] on the color histogram vectors and the Euclidean distance for the other descriptors, and they have been combined as specified in the original publication [5]. In particular, a relevance value is computed for each descriptor and the final score is calculated as a weighted linear combination.

For experimental purposes, a similar setup to that used in [5]7] has been employed. The available categories have been used as concepts, and user judgments about similarity have been simulated considering that only pictures under the same category represent the same concept. To simulate a search, a category is initially chosen at random. At each iteration, automatic judgments are made on the first 50 images, and submitted to the system. Then, the algorithm processes the selection and returns a new image ranking which is judged again as part of an iterative procedure.

To obtain more reliable data, each technique has been evaluated with 500 searches on each repository, using the same categories and initial picture order for all algorithms. In all cases, we have forced the situation that there is at least one relevant sample in between the first 50 images in the initial order in which pictures are presented.

Results have been measured in terms of precision at a cutoff value of 50 (the proportion of relevant picture amongst the top 50 ranked). These results are graphically shown in figure 3 .

The results show a significant improvement in retrieval precision when using the proposed technique, incorporating both the moderation term and the local

\footnotetext{
${ }^{1}$ Available in http://kdd.ics.uci.edu/databases/CorelFeatures
} 


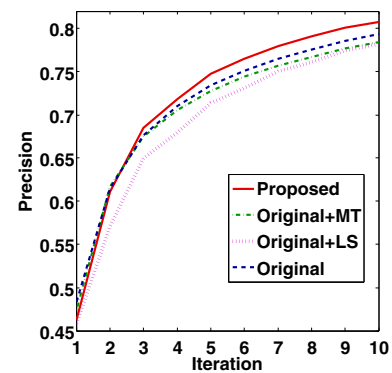

(a)

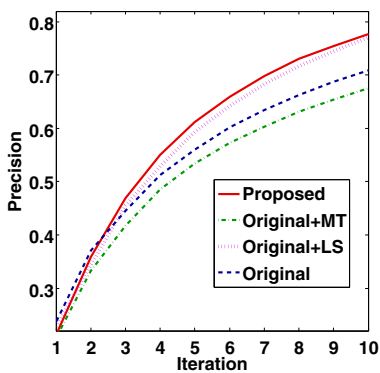

(b)

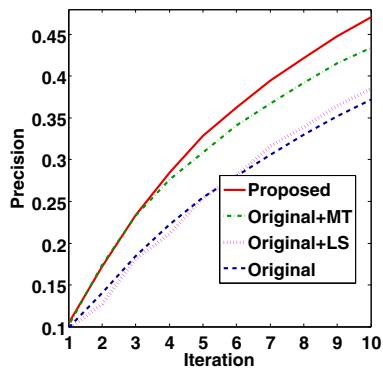

(c)

Fig. 3. Averaged retrieval precision measured on the first 50 retrieved images using a) Web, b) Corel, and c) Art databases

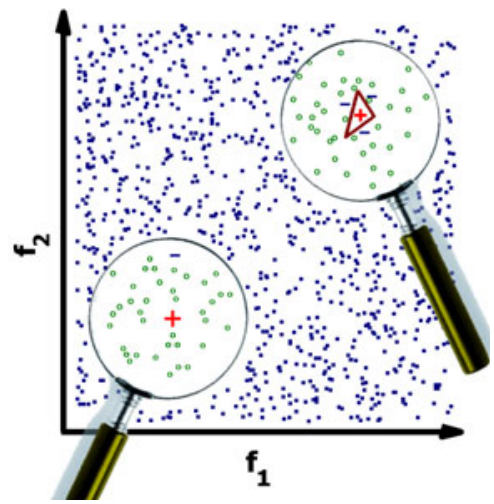

Fig. 4. When a local search strategy is adopted, the surrounded problem becomes more critical. Plus and minus signs represent samples that the user has marked as relevant and non relevant respectively, and each circle a picture in the database. The frontier delimits the area in which the expression $d_{N}\left(x_{i}\right) / d_{R}\left(x_{i}\right)$ yields a value greater than one, when a local search with respect to the relevant selection at the top right corner is performed.

searches approaches. Surprisingly enough, when these are used in isolation, worse results are obtained in some cases.

This is mainly due to the tight relation between the solutions to the two problems that we aim to solve. When only the local search strategy is adopted, the surrounding problem becomes more critical. This can easily be understood by considering the case illustrated in Figure 4. In this example, the original algorithm would not retrieve any of the pictures surrounding the positive selection at the top right corner (except the selection itself). However, if two local searches are performed and their results combined, half of the images retrieved (the ones which correspond to the relevant selection at the top right corner) will be those which yield a value of $d_{N}\left(x_{i}\right) / d_{R}\left(x_{i}\right)$ just below 1 (the farthest from positive 
and negative samples), very unlikely to be of any interest. Intuitively, neither option is correct. It is only when the moderation term is also introduced that pictures around the two positive selection are retrieved.

Similarly, if only the moderation term is introduced, the problem about differently populated areas becomes more noticeable. In the original technique, the larger effect of negative selections usually limits the amount of pictures with a large score around a single selection, helping diversity and playing a regulating effect with respect to density. In this sense, the reduction of the importance of non relevant selections caused by the moderating term may have a negative impact on the performance of the algorithm.

It is worth noting that the original NN approach may give even better results at the first and second iterations, e.g. on the Corel database. It is as the relevance feedback iterations progress that the improvements introduced become more and more important in all cases. The proposed method obtains the best results consistently and by a significant difference from the 4th iteration on. The only not significant difference is on Corel database and between the proposed method and the basic NN approach with local searches.

\section{Concluding Remarks}

We have presented an improved nearest neighbor based algorithm for CBIR. In particular, we have re-formulated the algorithm presented in [5] to make it more robust to differences in the densities of pictures in the feature space and the cardinality of the sets of relevant and non relevant selections. It has been observed that this new formulation allows for a significant increase in retrieval precision with respect to the original approach.

Note that in the formulation of the approach we have made no assumption on the feature space, the distance functions used for retrieval and the method used to combine these functions. Although a simple linear combination of the scores obtained for each descriptor has been used in the experimentation (as in [5]) and the use of a dissimilarity space has been suggested in [7, other strategies are also possible. In particular, combination methods which allow one to construct a single similarity measure from several distance functions (e.g. [17]) have been proposed. The integration of the nearest neighbor method with such approaches would make it possible to compute a single relevance score for each image by direct application of equation 2.

\section{References}

1. Datta, R., Joshi, D., Li, J., Wang, J.Z.: Image retrieval: Ideas, influences, and trends of the new age. ACM Computing Surveys 40(2), 1-60 (2008)

2. Lew, M.S., Sebe, N., Djeraba, C., Jain, R.: Content-based multimedia information retrieval: State of the art and challenges. ACM Transactions on Multimedia Computing, Communications and Applications 2(1), 1-19 (2006) 
3. Smeulders, A., Santini, S., Gupta, A., Jain, R.: Content-based image retrieval at the end of the early years. IEEE transactions on Pattern Analysis and Machine Intellingence 22(12), 1349-1379 (2000)

4. Zhou, X., Huang, T.: Relevance feedback for image retrieval: a comprehensive review. Multimedia systems 8(6), 536-544 (2003)

5. Giacinto, G., Roli, F.: Nearest-prototype relevance feedback for content based image retrieval. In: ICPR 2004: Proceedings of the Pattern Recognition, 17th International Conference on (ICPR 2004), Washington, DC, USA, vol. 2, pp. 989-992. IEEE Computer Society, Los Alamitos (2004)

6. Giacinto, G., Roli, F.: Instance-based relevance feedback for image retrieval. In: Saul, L.K., Bottou, Y.W. (eds.) Advances in Neural Information Processing Systems, vol. 17, pp. 489-496. MIT, Cambridge (2005)

7. Giacinto, G.: A nearest-neighbor approach to relevance feedback in content based image retrieval. In: Proceedings of the 6th ACM international conference on Image and video retrieval (CIVR 2007, pp. 456-463. ACM Press, Amsterdam (2007)

8. Ciocca, G., Schettini, R.: A relevance feedback mechanism for content-based image retrieval. Information processing and management 35(1), 605-632 (1999)

9. Urban, J., Jose, J.M.: Evidence combination for multi-point query learning in content-based image retrieval. In: ISMSE 2004: Proceedings of the IEEE Sixth International Symposium on Multimedia Software Engineering, pp. 583-586. IEEE Computer Society, Washington (2004)

10. Dasarathy, B. (ed.): Nearest Neighbor (NN) Norms: NN Pattern Classification Techniques. IEEE Computer Society Press, Los Alamitos (1991)

11. Shakhnarovich, G., Darrell, T., Indyk, P.: Nearest-Neighbor Methods in Learning and Vision: Theory and Practice. In: Neural Information Processing. The MIT Press, Cambridge (2006)

12. Duda, R.O., Hart, P.E., Stork, D.G.: Pattern Classification, 2nd edn. WileyInterscience, Hoboken (2000)

13. Paredes, R., Vidal, E.: Learning prototypes and distances: A prototype reduction technique based on nearest neighbor error minimization. Pattern Recognition 39(2), 180-188 (2006)

14. Barandela, R., Valdovinos, R., Sanchez, J., Ferri, F.: The imabalanced training sample problem: Under or over sampling? In: Fred, A., Caelli, T.M., Duin, R.P.W., Campilho, A.C., de Ridder, D. (eds.) SSPR\&SPR 2004. LNCS, vol. 3138, pp. 806-814. Springer, Heidelberg (2004)

15. Soille, P.: Morphological Image Analysis: Principles and Applications. Springer, Berlin (2003)

16. Swain, M.J., Ballard, D.H.: Color indexing. Int. J. Comput. Vision 7(1), 11-32 (1991)

17. Arevalillo-Herráez, M., Domingo, J., Ferri, F.J.: Combining similarity measures in content-based image retrieval. Pattern Recognition Letters 29(16), 2174-2181 (2008) 